\title{
A solar tornado caused by flares
}

\author{
N. K. Panesar, ${ }^{1,3}$ D. E. Innes, ${ }^{1}$ S. K. Tiwari, ${ }^{1}$ and B. C. Low ${ }^{2}$ \\ ${ }^{1}$ Max-Planck Institut für Sonnensystemforschung, Max-Planck-Str. 2, 37191, \\ Katlenburg-Lindau \\ email: panesar, innes, tiwari@mps.mpg.de \\ ${ }^{2}$ High Altitude Observatory, National Center for Atmospheric Research, \\ P.O. Box 3000, Boulder, CO 80307, USA \\ email: low@ucar.edu \\ ${ }^{3}$ Institut für Astrophysik, Georg-August-Universität Göttingen, Friedrich-Hund-Platz 1, \\ D-37077 Göttingen
}

\begin{abstract}
An enormous solar tornado was observed by SDO/AIA on 25 September 2011. It was mainly associated with a quiescent prominence with an overlying coronal cavity. We investigate the triggering mechanism of the solar tornado by using the data from two instruments: SDO/AIA and STEREO-A/EUVI, covering the Sun from two directions. The tornado appeared near to the active region NOAA 11303 that produced three flares. The flares directly influenced the prominence-cavity system. The release of free magnetic energy from the active region by flares resulted in the contraction of the active region field. The cavity, owing to its superior magnetic pressure, expanded to fill this vacated space in the corona. We propose that the tornado developed on the top of the prominence due to the expansion of the prominence-cavity system.
\end{abstract}

Keywords. Sun, prominences, cavity, corona and flares

\section{Introduction}

Solar prominences are composed of cool plasma, embedded in the hotter solar corona (Tandberg-Hanssen 1995). They typically form above the magnetic polarity inversion line (Martin 1973). They are known as prominences when seen above the solar limb and filaments, when observed on the solar surface (Zirker 1989; Mackay et al. 2010). Quiescent prominences are often seen with large-scale coronal cavities (Hudson et al. 1999; Gibson \& Fan 2006). Prominences sometimes appear to have rotating flows that resemble a tornado. Such prominences are known as prominence tornadoes (Pettit 1925, 1932). According to Pettit (1932) tornadoes appear like tightly wound flux ropes or vertical open spirals.

A huge quiescent-prominence tornado was observed by the Atmospheric Imaging Assembly (AIA) onboard the Solar Dynamic observatory (SDO) (Lemen et al. 2012) on 25 September 2011 ( $\mathrm{Li}$ et al. 2012; Panesar et al. 2013). Observations showed the flow of plasma along a helical structure and tornado-like rotations that lasted for more than 3 hours. We have analysed the tornado-like prominence and found that the acceleration of the tornado was correlated in time with the flares taking place in a neighbouring active region (Panesar et al. 2013).

In this paper, we show the relationship between the flares and the prominence. For this work, we have used the data from two different spacecraft - SDO/AIA and Solar TErrestrial RElations Observatory (STEREO)/ Extreme UltraViolet Imager (EUVI). We combine the observations from the two different directions and reconstruct the 3D geometry to study the tornado event. In the next section, we describe the observations and then discuss the results. 

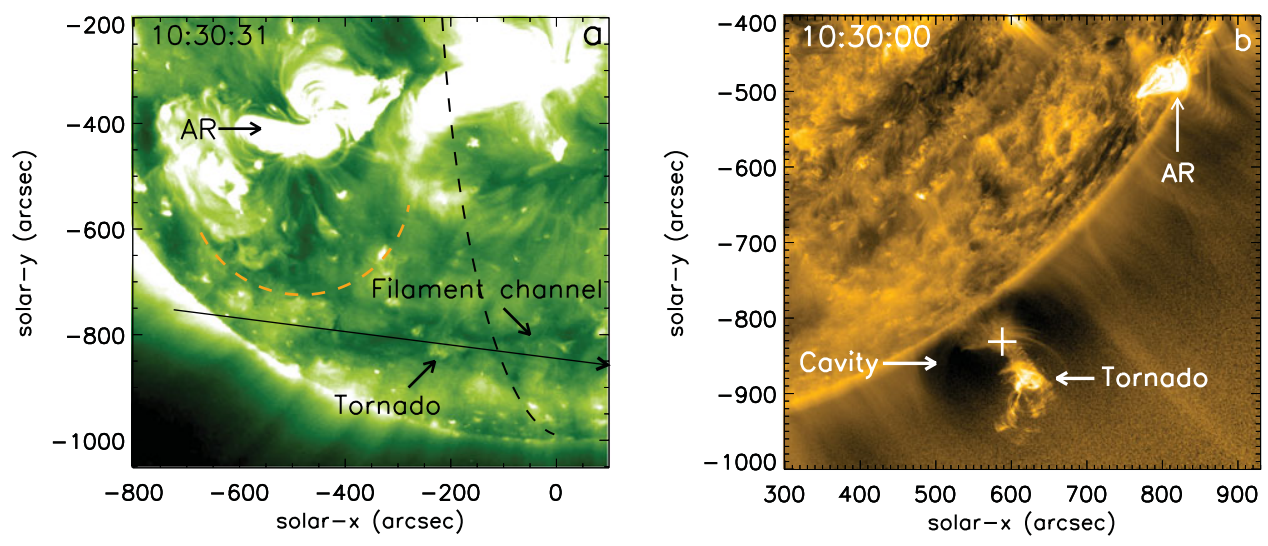

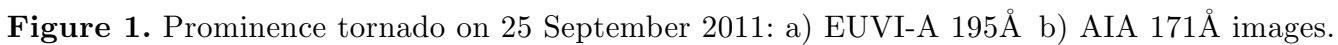
In (a) the black dashed line shows the position of SDO limb line on the EUVI-A image. The orange dashed line highlights the southern edge of the active region corona and the black long arrow is the epipolar line for the prominence position which is marked as a ' + ' sign on AIA image. The active region (11303) is indicated with an arrow on both images. (Image adapted from Panesar et al., A\&A, 549, A105, 2013, reproduced with permission (c) ESO).

\section{Observations}

The prominence was observed by the SDO/AIA on the south-west solar limb. It appeared near the disk center in STEREO/EUVI-A. The separation angle was $103^{\circ}$ between both the spacecrafts. For studying the limb perspective, we have used the SDO/AIA $171 \AA$ full-disk images of $12 \mathrm{~s}$ cadence. STEREO/EUVI-A $195 \AA$ images with a time cadence of 5 min have been used for the study of the filament on the solar disk.

Fig. 1 shows the overview of the prominence/filament system from two different angles - STEREO/EUVI-A and SDO/AIA. The active region (AR) and tornado are indicated with an arrows. We confirm the coordinates of the filament channel on disk by plotting the SDO limb line on STEREO images (black dashed line in Fig. 1a). The + sign on the prominence stem represents the position of selected coordinates along the long black arrow (epipolar line) on the EUVI image. The bright head of the tornado coincides with the epipolar line in the filament channel. The orange dashed line highlights the southern edge of the active region, which is only at a distance of $\sim 50$ arcsec from the filament channel.

There were three M-class flares from the active region at 02:45, 07:00 and 09:40 UT. All three flares were associated with CMEs and EUV waves. The third flare was the strongest and produced the most visible EUV wave front. Fig. 2 shows the $195 \AA$ running ratio image during the third flare. The faint EUV wave front swept over the filament channel about half an hour after the flare. After each flare, we noticed increased activity in the prominence. After the third flare there was accelerated tornado-like motion on the top of the prominence.

To investigate the relation between the flares and prominence dynamics, we used SDO limb images. We observed that the wave buffeted the prominence cavity system for all the flares. After the first flare, clear oscillations have been seen in the prominence system for about one hour (see Figure 7a of Panesar et al. (2013)). The second flare was at 06:45 UT, at the time of an SDO eclipse. Since this was an important time for the tornado activity, we used SWAP data to see the relation between the second flare and the tornado activation. The SWAP observations clearly show that the increase in prominence activity 


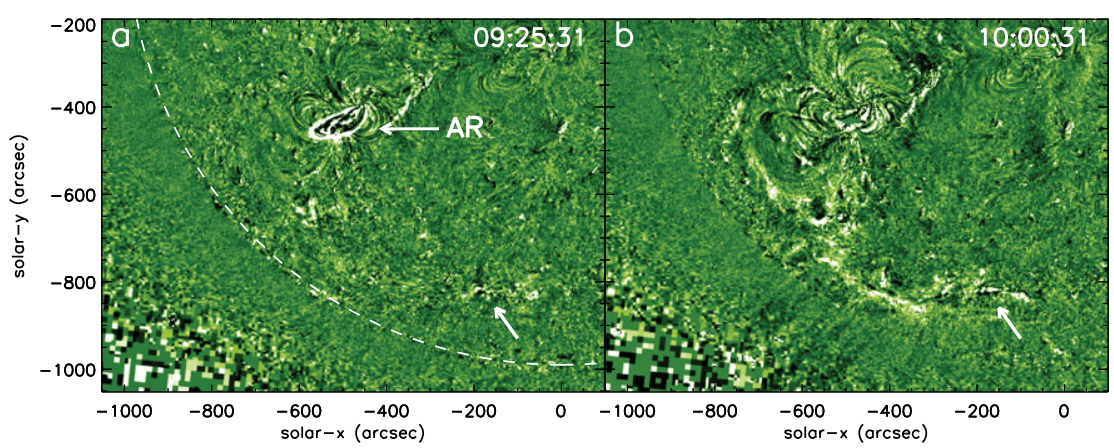

Figure 2. STEREO/EUVI-A $195 \AA$ running ratio images. a) The onset of the third flare b) is associated with an EUV wave. The dashed line is the solar limb line and the small arrows point to the tornado site.

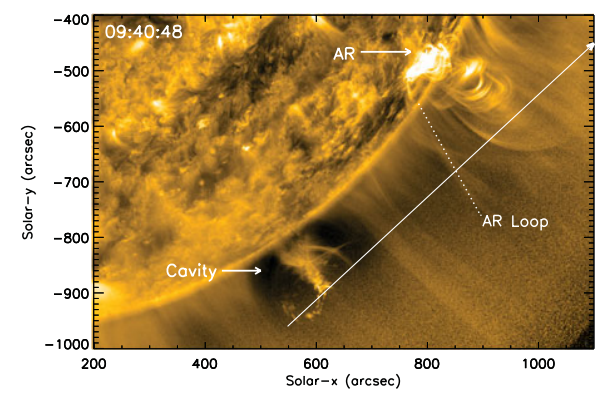

Figure 3. SDO/AIA $171 \AA$ intensity image. The long arrow shows the position of time-series in Fig. 4 taken through the prominence, cavity, active region loop (dotted line) and active region.

after the second flare. We noticed from the SDO images, that there was an extension of the prominence towards the active region after the second flare.

The main change occurred in the cavity after the third flare. Fig. 4 shows the time-series along the diagonal arrow in Fig. 3. The flare started at 09:20 UT and the active region loop started to move southward just after that. This resulted in a sharp contraction in the cavity boundary (indicated by an arrow in Fig. 4). The cavity contracted about 10 $\mathrm{Mm}$ in width. After that the active region loop swayed back towards the active region. Then the cavity started to expand in the direction of the neighbouring active region. The cavity grew in size from $140 \mathrm{Mm}$ to $167 \mathrm{Mm}$ in 3.5 hours. At the same time, the prominence also grew in size (see Fig. 4) and developed the tornado at the top.

\section{Discussion}

A huge solar tornado was observed by SDO/AIA on the SW solar limb and by STEREO/EUVI-A at disk center, on 25 September 2011. We noticed from the SDO/AIA images, that there was an active region near to the prominence-cavity system, which produced three flares on 25 September. We suspected that the flares influenced the prominence-cavity system. A careful analysis of STEREO/EUVI-A images showed that all three flares were associated with CMEs and EUV waves. EUV waves swept over the filament channel and increased the prominence activity. A tornado developed after the third flare.

After the first flare, there were oscillations in the prominence main body. The second flare caused an arm-like extension of the prominence towards the active region. The biggest change occurred after the third flare. It influenced the surrounding corona and the neighbouring cavity. During the third flare, the active region loop started to expand 


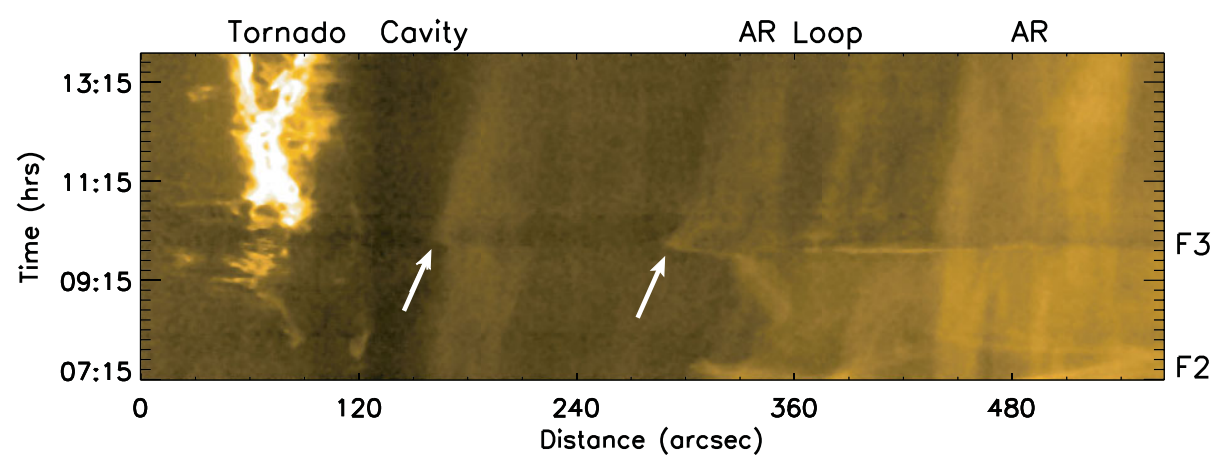

Figure 4. SDO/AIA $171 \AA$ intensity time-series image along the long diagonal arrow in Fig. 3 . F2 and F3 show the time of the second and third flare. The small white arrows point to the contraction in the cavity edge ( 09:40 UT) and expanding of the active region loop.

and move towards the edge of the cavity, which resulted in a sharp contraction in the cavity boundary, followed by an outward expansion in the cavity after the flare. The cavity continued visibly expanding for 3.5 hours. During this time the prominence increased in height and formed a tornado-like structure. It is likely that plasma moving along the helical field lines produced the tornado appearance.

We speculate that the expansion of the prominence was a result of the cavity expansion. We know that flares and CMEs are a means to release free magnetic energy (Zhang \& Low 2005), which can result in a contraction of the field lines at the flare site (Hudson 2000; Zhang \& Low 2003; Janse \& Low 2007). In the present scenario, the neighbouring cavity expanded towards the active region to fill the surrounding corona, that had contracted after the flare. The cavity expanded because it had a higher magnetic pressure than that on the flare site. Expansion of the cavity led to a decrease of the magnetic pressure inside the cavity which resulted in the expansion of the prominence field, and this led to the development of the tornado-like activity. A detailed mathematical model of this observation is given in Panesar et al. (2013).

\section{Acknowledgements}

We are obliged to the SDO and STEREO teams. The presentation of this paper in IAU symposium 300 was possible due to the partial support by IAU and DAAD.

\section{References}

Gibson, S. E. \& Fan, Y. 2006, Journal of Geophysical Research (Space Physics), 111, 12103

Hudson, H. S. 2000, ApJ, 531, L75

Hudson, H. S., Acton, L. W., Harvey, K. L., \& McKenzie, D. E. 1999, ApJ, 513, L83

Janse, Å. M. \& Low, B. C. 2007, A\&A, 472, 957

Kitiashvili, I. N., Kosovichev, A. G., et al. A. A. 2013, ApJ, 770, 37

Lemen, J. R., Title, A. M., Akin, D. J., et al. 2012, Solar Phys., 275, 17

Li, X., Morgan, H., Leonard, D., \& Jeska, L. 2012, ApJ, 752, L22

Mackay, D. H., Karpen, J. T., Ballester, J. L., et al. 2010, Space Sci. Rev., 151, 333

Martin, S. F. 1973, 31, 3

Panesar, N. K., Innes, D. E., Tiwari, S. K., \& Low, B. C. 2013, A\&A, 549, A105

Pettit, E. 1925, Publications of the Yerkes Observatory, 3, 4

Pettit, E. 1932, ApJ, 76, 9

Tandberg-Hanssen, E., ed. 1995, ASSL, Vol. 199, The nature of solar prominences

Zhang, M. \& Low, B. C. 2003, ApJ, 584, 479

Zhang, M. \& Low, B. C. 2005, ARA\&A, 43, 103

Zirker, J. B. 1989, Solar Phys., 119, 341 